\title{
A Hybrid Multi-Strategy Recommender System Using Linked Open Data
}

\author{
Petar Ristoski ${ }^{1}$ and Eneldo Loza Mencía ${ }^{2}$ and Heiko Paulheim ${ }^{1}$ \\ 1 University of Mannheim, Germany \\ Research Group Data and Web Science \\ \{petar.ristoski, heiko\}@informatik. uni-mannheim.de \\ 2 Technische Universität Darmstadt \\ Knowledge Engineering Group \\ eneldo@ke.tu-darmstadt.de
}

\begin{abstract}
In this paper, we discuss the development of a hybrid multistrategy book recommendation system using Linked Open Data. Our approach builds on training individual base recommenders and using global popularity scores as generic recommenders. The results of the individual recommenders are combined using stacking regression and rank aggregation. We show that this approach delivers very good results in different recommendation settings and also allows for incorporating diversity of recommendations.
\end{abstract}

Keywords: Linked Open Data, Hybrid Recommender Systems, Stacking

\section{Overall Approach}

We propose a hybrid, multi-strategy approach that combines the results of different base recommenders and generic recommenders into a final recommendation. A base recommender is an individual collaborative or content based recommender system, whereas a generic recommender makes a recommendation solely on some global popularity score, which is the same for all users. For base recommenders, we use two collaborative filtering strategies (item and user based), as well as different content-based strategies exploiting various feature sets created from DBpedia ${ }^{1}$. Our results in the three tasks of the LOD-enabled Recommender Systems Challenge $2014^{2}$ demonstrate the effectiveness of our approach.

\section{Generic Recommenders}

We use different generic recommenders in our approach. First, the RDF Book Mashup dataset ${ }^{3}$ provides the average score assigned to a book on Amazon. Furthermore, DBpedia provides the number of ingoing links to the Wikipedia

${ }^{1}$ http://dbpedia.org

275,559 numeric ratings on 6,166 books (from 0-5, Task 1 ) and 72,372 binary ratings on 6733 books (Tasks 2 and 3), resp., from 6,181 users for training, and evaluation on 65,560 and 67,990 unknown ratings, resp. See http://challenges.2014.eswcconferences.org/index.php/RecSys for details.

${ }^{3}$ http://wifo5-03.informatik.uni-mannheim.de/bizer/bookmashup/ 
article corresponding to a DBpedia instance, and the number of links to other datasets (e.g., other language editions of DBpedia), which we also use as global popularity measures. Finally, SubjectiveEye3D delivers a subjective importance score computed from Wikipedia usage information ${ }^{4}$.

\section{Features for Content-based Recommendation}

The features for content-based recommendation were extracted from DBpedia using the RapidMiner Linked Open Data extension [2]. We use the following feature sets for describing a book:

- All direct types of a book ${ }^{5}$

- All categories of a book

- All categories of a book's author(s)

- All categories of a book including broader categories ${ }^{6}$

- All categories of a book's author(s) and of all other books by the book's authors

- All genres of a book and of all other books by the book's authors

- All authors that influenced or were influenced by the book's authors

- A bag of words created from the abstract of the book in DBpedia. That bag of words is preprocessed by tokenization, stemming, removing tokens with less than three characters, and removing all tokens less frequent than $3 \%$ or more frequent than $80 \%$.

Furthermore, we created a combined book's feature set, comprising direct types, qualified relations, genres and categories of the book itself, its previous and subsequent work and the author's notable work, the language and publisher, and the bag of words from the abstract. Table 1 depicts the number of features in each set.

Besides DBpedia, we made an effort to retrieve additional features from two additional LOD sources: British Library Bibliography and DBTropes ${ }^{7}$. Using the RapidMiner LOD extension, we were able to link more than $90 \%$ of the books to BLB entities, but only $15 \%$ to DBTropes entities. However, the generated features from BLB were redundant with the features retrieved from DBpedia, and the coverage of DBTropes was too low to derive meaningful features. Hence, we did not pursue those sources further.

\section{Recommender Strategies}

For implementing the collaborative and content-based recommendation systems, we used the RapidMiner Recommendation Extension [1], which uses k-NN classification. We use $k=80$ and cosine similarity for the base recommenders. The rationale of using cosine similarity is that, unlike, e.g., Euclidean distance, only

\footnotetext{
${ }^{4}$ https://github.com/paulhoule/telepath/wiki/SubjectiveEye3D

${ }^{5}$ This includes types in the YAGO ontology, which can be quite specific (e.g., American Thriller Novels)

6 The reason for not including broader categories by default is that the category graph is not a cycle-free tree, with some subsumptions being rather questionable.

${ }^{7}$ http://bnb.data.bl.uk/ and http://skipforward.opendfki.de/wiki/DBTropes
} 
common features influence the similarity, but not common absence of features (e.g., two books not being American Thriller Novels).

Furthermore, we train an additional recommender on the joint feature set, using Random Decision Trees (RDTs) [4]. RDTs generate $k_{1}$ decision trees with maximal depth $k_{2}$ and random attribute tests at the inner nodes. Each tree collects a distribution over the target variables at each of its leaf nodes by seeing the training data. The predictions of several of such trees are then combined into a final prediction. RDTs provide a good tradeoff between scalability for large example sets and prediction accuracy (often outperforming SVMs).

For applying RDTs to the collaborative filtering data, we transformed the problem into a multilabel task: For each user we generated $n$ different labels indicating each of the possible user ratings, i.e. $n=5$ for task 1 and $n=2$ for task 2. During training RDTs learn - for each known book/user combination - the mapping between the feature set of each book and the generated labels. Given an unknown book/user combination $x, y$, we are now able to estimate a distribution $P(i \mid x, y)$ over the different ratings $i$. The final predicted rating $r$ is obtained by weighting the ratings $r=\sum_{i=0}^{n} i \cdot P(i \mid x, y)$.

RDTs do not suffer from high dimensionality and sparseness as much as k$\mathrm{NN}$ does, thus we have built $k_{1}=10$ trees with depth $k_{2}=10$ on the combined book's properties feature set, instead of individual RDTs on each feature set. ${ }^{8}$

\section{$2 \quad$ Predicting Ratings and Top k Lists}

For predicting ratings (task 1 in the challenge), we use all the recommendation algorithms discussed above for training a regression model in the range of $[0,5]$. The results for the base recommenders are shown in Fig. 1.

In order to create a more sophisticated combination of the different base and generic recommenders, we trained a stacking model as described in [3]: We trained the base recommenders in 10 rounds in a cross validation like setting, collected their predictions, and learned a stacking model on the predictions. The results in Table 1 show that the stacked prediction outperforms the base and generic recommenders, with the RDT based stacking (with $k_{1}=500$ and $k_{2}=20$ ) slightly ahead of linear regression, and both stacking approaches outperforming the baseline approach of averaging all recommenders' ratings.

To further analyze the contribution of each feature, we also report the $\beta$ parameters found by linear regression. It can be observed that apart from the direct types, all base and generic recommenders contribute to the linear regression. A possible reason for that anomaly is that direct types and categories are rather redundant. Furthermore, we can see the benefit of using stacking approaches as the three recommenders with low RMSE are filtered out by the LR model.

For creating top $\mathrm{k}$ lists from binary ratings (task 2 in the challenge), we again trained regression models like for rating prediction, using a range of $[0 ; 1]$. The top $\mathrm{k}$ lists were then obtained by ranking by the predicted rating. As shown in

\footnotetext{
${ }^{8}$ In general, it holds that the higher $k_{1}$ and $k_{2}$ the better. We tried to find a good balance between computational costs and predictive quality.
} 
Table 1: Performances of the base and generic recommenders, the number of features used for each base recommender, and the performance of the combined recommenders

\begin{tabular}{|c|c|c|c|c|}
\hline & & \multicolumn{2}{|c|}{ Task 1} & Task 2 \\
\hline Recommender & \#Features & RMSE & LR $\beta$ & F-Score \\
\hline Item-based collaborative filtering & - & 0.8843 & +0.269 & 0.5621 \\
\hline User-based collaborative filtering & - & 0.9475 & +0.145 & 0.5483 \\
\hline Book's direct types & 534 & 0.8895 & -0.230 & 0.5583 \\
\hline Author's categories & 2,270 & 0.9183 & +0.098 & 0.5576 \\
\hline Book's (and author's author books') genres & 582 & 0.9198 & +0.082 & 0.5567 \\
\hline Combined book's properties & 4,372 & 0.9421 & +0.0196 & 0.5557 \\
\hline Author and influenced/influencedBy authors & 1,878 & 0.9294 & +0.122 & 0.5534 \\
\hline Books' categories and broader categories & 1,987 & 0.939 & +0.012 & 0.5509 \\
\hline Abstract bag of words & 227 & 0.8893 & +0.124 & 0.5609 \\
\hline RDT recommender on combined book's properties & 4,372 & 0.9223 & +0.128 & 0.5119 \\
\hline Amazon rating & - & 1.037 & +0.155 & 0.5442 \\
\hline Ingoing Wikipedia links & - & 3.9629 & +0.001 & 0.5377 \\
\hline SubjectiveEye3D score & - & 3.7088 & +0.001 & 0.5369 \\
\hline Links to other datasets & - & 3.3211 & +0.001 & 0.5321 \\
\hline Average of all individual recommenders & 14 & 0.8824 & - & - \\
\hline Stacking with linear regression & 14 & 0.8636 & - & 0.4645 \\
\hline Stacking with RDT & 14 & 0.8632 & - & 0.4966 \\
\hline Borda rank aggregation & 14 & - & - & 0.5715 \\
\hline
\end{tabular}

Table 1, the base recommenders worked quite well, but the combination with linear regression delivered non-satisfying results. The reason is that the outcome of the base recommenders is not scaled equally for each user, but strongly depends on the user's total number of positive and negative ratings. This made it impossible to learn a suitable regression function.

However, we observed that despite being incompatible in scale, the base and generic recommenders delivered good rankings for each user. Thus, we performed an aggregation of the rankings produced by the different recommenders, using Borda's rank aggregation algorithm, which outperforms all the individual recommenders, as well as the stacking regression.

\section{Creating Diverse Predictions}

The final task in the challenge was to address diversity of predictions, i.e., trade off the accuracy of predictions, measured in F1 score, and their diversity, measured in intra-list diversity (ILD), both on a top $\mathrm{k}$ list. To address that trade-off, we followed a greedy top down approach which creates a ranking as for top $\mathrm{k}$ lists. First, we select the top $m$ items from that list. Then, we process the list from position $m+1$ on, adding each book that does not share author and categories with any of the books already on the list, until the list has $k$ items.

The results are depicted in Fig. 1 for $\mathrm{k}=20$, selecting items from a list of the top 100 predictions. It can be observed that the F1 score gradually rises when 


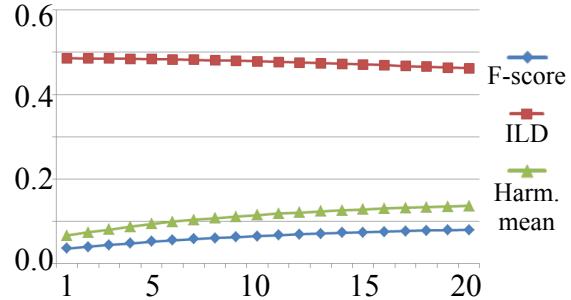

(a) F-measure, ILD and their harmonic mean ( $y$-axis) for different values of $m$

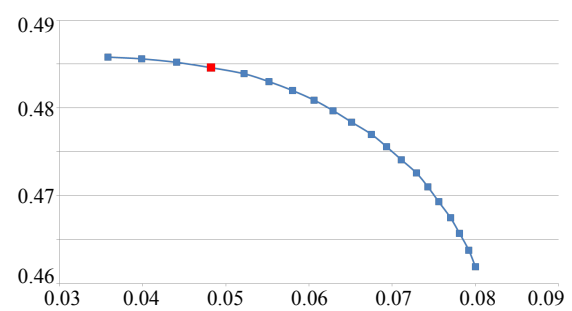

(b) ILD (y-axis) plotted against Fmeasure ( $x$-axis)

Fig. 1: Trade-off between F-measure and diversity

using higher values of $m$, while the ILD drops. Although the harmonic is optimal for using simply the top 20 predictions (given the different orders of magnitude of F1 and ILD), we decided to submit the solution with $m=4$ to the challenge. ${ }^{9}$

\section{Conclusion and Outlook}

In this paper, we have layed out a hybrid multi-strategy approach for linked data enabled recommender systems. We have shown that combining the predictions of different base recommenders is a feasible strategy, and that generic (i.e., non user specific) recommenders can be a useful ingredient.

In particular, our approach allows for the addition of new feature groups without interaction effects, and for the combination of different recommender strategies. By exploiting stacking regression, an optimal combination of different recommenders can be found automatically, however, for ranking-based problems, rank aggregation turned out to be the more promising strategy.

\section{Acknowledgements}

The work presented in this paper has been partly funded by the German Research Foundation (DFG) under grant number PA 2373/1-1 (Mine@LOD).

\section{References}

1. M. Mihelčić, N. Antulov-Fantulin, M. Bošnjak, and T. Šmuc. Extending rapidminer with recommender systems algorithms. In RapidMiner Community Meeting and Conference (RCOMM 2012), 2012.

2. H. Paulheim and J. Fürnkranz. Unsupervised Generation of Data Mining Features from Linked Open Data. In International Conference on Web Intelligence, Mining, and Semantics (WIMS'12), 2012.

3. K. M. Ting and I. H. Witten. Issues in stacked generalization. Artificial Intelligence Research, 10(1), 1999.

4. X. Zhang, Q. Yuan, S. Zhao, W. Fan, W. Zheng, and Z. Wang. Multi-label classification without the multi-label cost. In Proceedings of the 2010 SDM, 2010.

\footnotetext{
${ }^{9}$ The reason is that the challenge uses the average rank w.r.t. F1 and ILD as a scoring function, which makes the selection of an optimal parameter strongly depend on the other participants' solutions. It turned out that $m=4$ optimized our scoring.
} 